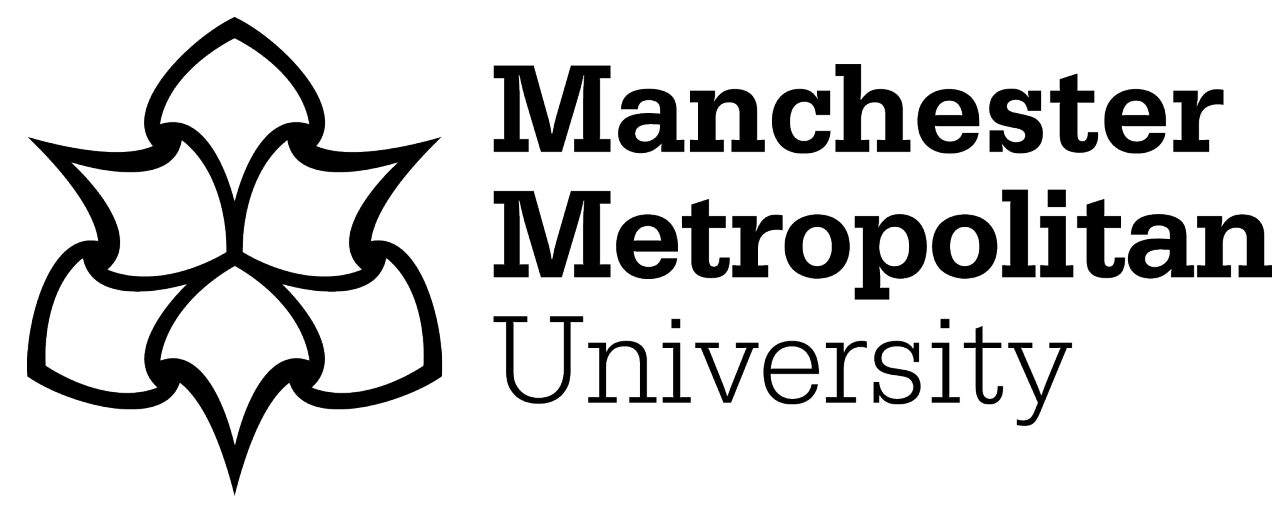

Antonides, Lysbeth H, Brignall, Rachel M, Costello, Andrew, Ellison, Jamie, Firth, Samuel E, Gilbert, Nicolas, Groom, Bethany J, Hudson, Samuel J, Hulme, Matthew C, Marron, Jack, Pullen, Zoe A, Robertson, Thomas BR, Schofield, Christopher J, Williamson, David C, Kemsley, E Kate, Sutcliffe, Oliver B ORCID logoORCID: https://orcid.org/0000-0003-3781-7754 and Mewis, Ryan E ORCID logoORCID: https://orcid.org/0000-0002-37566505 (2019) Rapid Identification of Novel Psychoactive and Other Controlled Substances Using Low-Field 1H NMR Spectroscopy. ACS Omega, 4 (4). pp. 7103-7112. ISSN 2470-1343

Downloaded from: https://e-space.mmu.ac.uk/622850/

Version: Published Version

Publisher: American Chemical Society (ACS)

DOI: https://doi.org/10.1021/acsomega.9b00302

Usage rights: Creative Commons: Attribution-Noncommercial-No Derivative Works 4.0

Please cite the published version 


\section{Rapid Identification of Novel Psychoactive and Other Controlled Substances Using Low-Field ${ }^{1} \mathrm{H}$ NMR Spectroscopy}

Lysbeth H. Antonides, ${ }^{\dagger, \#}$ Rachel M. Brignall, ${ }^{\ddagger}$ Andrew Costello, ${ }^{\S, \perp}$ Jamie Ellison, ${ }^{\dagger, \S, \perp}$ Samuel E. Firth, ${ }^{\dagger}$ Nicolas Gilbert, ${ }^{\dagger, \perp}$ Bethany J. Groom, ${ }^{\dagger}$ Samuel J. Hudson, ${ }^{\dagger}$ Matthew C. Hulme, ${ }^{\dagger, \perp}$ Jack Marron, ${ }^{\dagger, \perp}$ Zoe A. Pullen, ${ }^{\dagger}$ Thomas B. R. Robertson, ${ }^{\dagger}$ Christopher J. Schofield, ${ }^{\S, \perp}$ David C. Williamson, ${ }^{*},{ }^{\ddagger}$

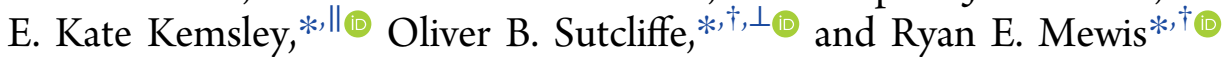

${ }^{\dagger}$ School of Science and the Environment, Division of Chemistry and Environmental Science, Manchester Metropolitan University, John Dalton Building, Chester Street, Manchester M1 5GD, U.K.

‡Oxford Instruments, Tubney Woods, Abingdon, Oxfordshire OX13 5QX, U.K.

${ }^{\S}$ Greater Manchester Police, Openshaw Complex, Lawton Street, Openshaw, Manchester M11 2NS, U.K.

"Quadram Institute Bioscience, Norwich Research Park, Norwich NR4 7UA, U.K.

${ }^{\perp}$ MANchester DRug Analysis and Knowledge Exchange (MANDRAKE), Manchester Metropolitan University, John Dalton Building, Chester Street, Manchester M1 5GD, U.K.

Supporting Information
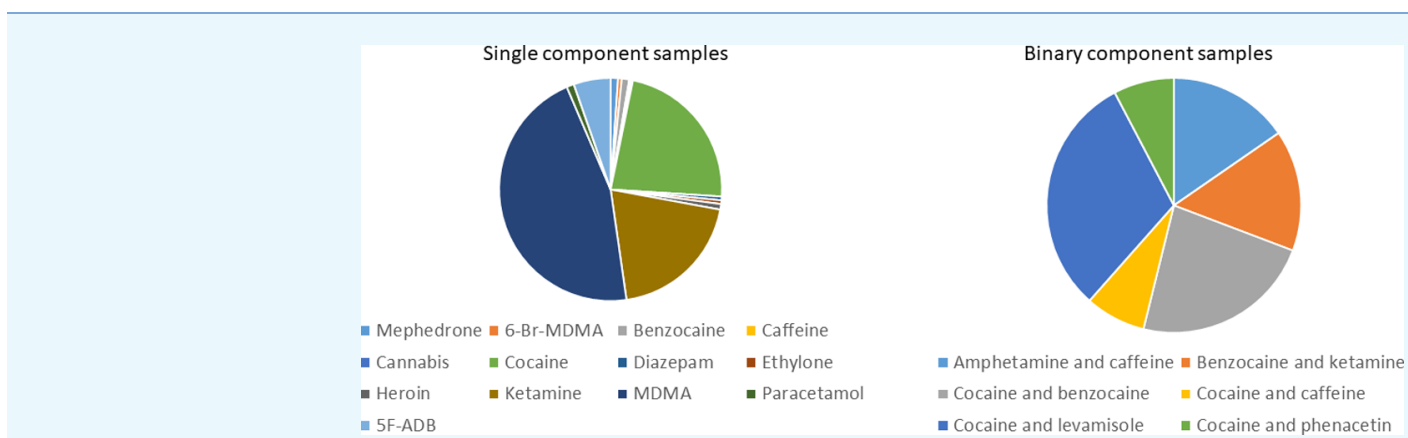

ABSTRACT: An automated approach to the collection of ${ }^{1} \mathrm{H}$ NMR (nuclear magnetic resonance) spectra using a benchtop NMR spectrometer and the subsequent analysis, processing, and elucidation of components present in seized drug samples are reported. An algorithm is developed to compare spectral data to a reference library of over $300{ }^{1} \mathrm{H}$ NMR spectra, ranking matches by a correlation-based score. A threshold for identification was set at 0.838 , below which identification of the component present was deemed unreliable. Using this system, 432 samples were surveyed and validated against contemporaneously acquired GC-MS (gas chromatography-mass spectrometry) data. Following removal of samples which possessed no peaks in the GC-MS trace or in both the ${ }^{1} \mathrm{H}$ NMR spectrum and GC-MS trace, the remaining 416 samples matched in $93 \%$ of cases. Thirteen of these samples were binary mixtures. A partial match (one component not identified) was obtained for $6 \%$ of samples surveyed whilst only $1 \%$ of samples did not match at all.

\section{INTRODUCTION}

The detection, and identification, of drugs within a seized sample is a desirable means of evidence and intelligence gathering. ${ }^{1}$ Given the growing issue of novel psychoactive substances (NPS) and herbal products mixed with synthetic cannabinoids, known as spice, ${ }^{2,3}$ a technique is required for the detection of the active pharmaceutical ingredient (API) within a seized sample, if indeed it is present. Such a technique is urgently needed, given that the number of NPS reported to the European Monitoring Centre for Drugs and Drug Addiction (EMCDDA) has risen from 13 in 2008 to 101 in $2014^{4}$ and that in 2016, a survey of 625 UK inmates reported $33 \%$ had used spice in the last month. ${ }^{5}$

The established analytical tools of GC-MS (gas chromatography-mass spectrometry) and HPLC-MS (high perform- ance liquid chromatography-MS), which are employed by forensic laboratories (and by harm reduction point-of-care services $^{6}$ ) for the identification and quantification of drugs of abuse, struggle with the high-throughput that is required to test the number of seized samples. Additionally, in some instances, the reference standards are not available for comparison. Recently, a number of studies have been reported that focus on the use of various spectroscopic techniques to achieve high sample throughput, whilst also detecting the API present in seized samples. Studies have utilized IR (infra-red) spectros-

Received: February 1, 2019

Accepted: March 19, 2019

Published: April 19, 2019 
copy, ${ }^{7-10}$ Raman spectroscopy, ${ }^{11-14}$ NMR (nuclear magnetic resonance), ${ }^{9,10,15-17}$ HPLC, ${ }^{9,10,18-21}$ and GC-MS.,10,22-25

IR and Raman spectroscopies have been used for the analysis of more than 200 seized NPS samples, resulting in the identification of a single component or a mixture in $76 \%$ of the samples surveyed. ${ }^{26} 17 \%$ of samples were partially identified, with a further $7 \%$ that were unidentifiable. One of the limitations of this study was the lack of reference spectra; the reference library needed to be extended through the independent identification of NPS via NMR and MS.

A recent development concerning Raman spectroscopy is the use of a portable hand-held technology for mobile detection. A study employing 785 and $1064 \mathrm{~nm}$ excitation sources highlighted that the latter wavelength was more suitable, giving fewer false positives and false negatives for the NPS screened whilst also significantly lowering fluorescence. ${ }^{27}$ In addition, IR has been used in conjunction with chemometric methods (partial least squares (PLS) discriminant analysis) to distinguish four classes of drugs with an average reliability rate of $98.6 \%$ for the 932 samples surveyed. ${ }^{8} \mathrm{~A}$ further use of PLS-based analysis in conjunction with Raman and IR spectroscopies has been exemplified for the quantification of cocaine in samples consisting of cocaine, sodium carbonate, and either caffeine or lidocaine. ${ }^{28}$

A further study utilized liquid chromatography coupled to high-resolution MS for the detection of NPS in wastewater from a festival in Amsterdam. ${ }^{29}$ Implementation of an algorithm to extract accurate masses which were then compared to an in-house database of 2000 entries of NPS and transformation products facilitated the detection of eight NPS compounds.

The combination of dopant-assisted positive photoionization ion mobility spectrometry (DAPP-IMS) and direct analysis in real time time-of-flight mass spectrometry (DART-TOF MS) has been employed successfully to screen drug samples with minimal sample preparation. ${ }^{30}$ Although the method provided a fast response and high-throughput, the method was limited due to its inability to identify all the components in a mixture or unambiguously identify similar compounds, such as epimers.

A method utilizing GC-MS for the analysis of cathinones, amphetamine-like stimulants, and other NPS has been developed that incorporates an almost simultaneous derivatization and extraction step, which is achieved through the use of hexyl chloroformate and dispersive liquid-liquid microextraction. $^{22}$ The chromatographic method allowed the separation of 26 stimulants, which were all resolved in under $15 \mathrm{~min}$.

The development of a compound database and HRMS (high-resolution mass spectrometry) spectral library, consisting of data for 875 and 252 NPS from multiple classes, respectively, has been utilized for the analysis of 13 serum samples that had been spiked with up to five NPS per sample. $^{31} 32$ of the 35 compounds present were detected using the method developed. Furthermore, HRMS-based screening of four seized samples provided results consistent with official forensic laboratory findings.

NMR, despite being a powerful analytical technique, has been underutilized for the detection of drugs within samples. This is largely because of the perceived complexity of the measurement but also the inherent cost of requiring a superconducting magnet and the associated technical costs. However, the recent development of bench-top NMR spectrometers has made this technique a much more attractive and affordable prospect. This approach is exemplified through the identification of drugs in real case samples, carried out for two samples, using a limited library of twelve spectra on a benchtop $80 \mathrm{MHz}$ spectrometer and compared by visual inspection with data collected on a $600 \mathrm{MHz}$ spectrometer. ${ }^{32}$ Another report has investigated the differentiation of 65 fentanyl analogues, by eye, using ${ }^{1} \mathrm{H}$ NMR spectra obtained on a low-field spectrometer $(62 \mathrm{MHz}){ }^{33}$ Quantum mechanical spin system analysis was employed in this report, and this highlighted that field strength-independent ${ }^{1} \mathrm{H}$ NMR libraries could be useful for drug analysis. Furthermore, the quantification of components in over-the-counter painkiller drugs has been utilized in an undergraduate practical, again using a visual inspection to identify the various components. ${ }^{16}$

Screening of spice samples using low-field NMR has been explored by Gilard and co-workers. ${ }^{34}$ In their study, nine synthetic cannabinoids were detected and quantified. Although high-field NMR spectroscopy, sometimes in conjunction with mass spectrometry, is required for unambiguous structural identification, low-field NMR provided useful insights, in the form of diagnostic signals, as to their chemical structure. However, the greater spectral band overlap compared to data acquired from a superconducting system did present a data analysis challenge.

In this paper, we detail a fully automated NMR system that acquires the ${ }^{1} \mathrm{H}$ NMR spectrum of a sample, processes it, and then using a developed pattern recognition algorithm, compares the spectrum to a reference library of over $300{ }^{1} \mathrm{H}$ NMR spectra, before finally reporting the $\operatorname{drug}(\mathrm{s})$ present (if there are any to detect). The simplistic nature of the experimental measurement overcomes the need for dedicated technical staff, and the robustness of the algorithm for spectral analysis means that it can be readily utilized in custodial suites.

\section{RESULTS AND DISCUSSION}

Reference Library and Algorithm Development. ${ }^{1} \mathrm{H}$ NMR spectra of all reference compounds were collected using the Pulsar instrument. The reference library thus consisted of 302 spectra altogether. The large number and types of materials represented in the reference library enable a wide range of seized materials to be identified and also allow for various tests of the performance of the pattern recognition algorithm.

The ${ }^{1} \mathrm{H}$ NMR spectra were truncated into two discrete sections: the "class" region $(0.46-1.54 \mathrm{ppm})$ and a fingerprint region (3.90-12.50 ppm). "Classes" of compounds possess similar backbone structures, and hence spectral features, but it was imperative that the algorithm developed could also differentiate compounds within a class. Both sections were used in the pattern recognition process. Further, to account for some variations in the dimethyl sulfoxide (DMSO) signal that was used as an internal standard, the algorithm accommodated a tolerance of $\pm 0.06 \mathrm{ppm}$ on the chemical shift of any peak.

Before commencing any analysis of seized samples, the algorithm's performance in terms of correct identifications was tested by using it to the validation sample spectra. This allowed evaluation of technical repeatability, as well as the ability of the method to detect and identify previously unseen mixtures and an estimation of the rate of false-positives.

Of the 130 controlled substance replicates, the class was correctly identified in all but one of the samples ( $>99 \%$ success rate) and the compound in all but six ( $>95 \%$ success rate). 
<smiles>COC(=O)C1C2CCC(C2)N1COC(=O)c1ccccc1</smiles>

Cocaine

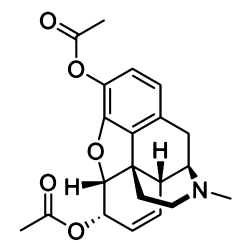<smiles>CCNC(C)C(=O)c1ccc2c(c1)OCO2</smiles><smiles>CNC(C)Cc1ccc2c(c1)OCO2</smiles>

MDMA<smiles>CNC1(c2ccccc2Cl)CCCCC1=O</smiles>

Ketamine<smiles>CC(=O)Nc1ccc(O)cc1</smiles>

Paracetamol<smiles>CNC(C)C(=O)c1ccc(CNC(C)Cc2cc3c(cc2Br)OCO3)cc1</smiles>

6-Br-MDMA

Mephedrone

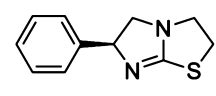

Levamisole

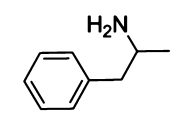

Amphetamine<smiles>CN1C(=O)C2C(NCN2C)N(C)C1=O</smiles>

Phenacetin<smiles>CCCCCCCCCC(=O)Nc1ccc(OCC)cc1</smiles><smiles>CNCCc1cccs1</smiles>

2-aminoindane

Methiopropamine

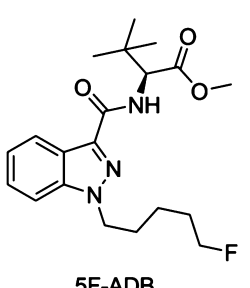

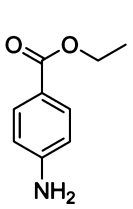

Benzocaine<smiles>Cc1ccc2c(c1)C(c1ccccc1)NCC(=O)N2C</smiles>

Diazepam

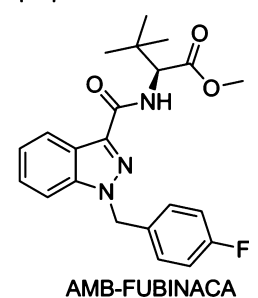

Figure 1. Compounds detected in the seized samples as part of this work.

Notably, all of these were from the diphenidine class, of which 5 were misidentified as other closely related diphenidines. Note that the diphenidines were somewhat over-represented amongst the technical replicates due to the ready availability of these compounds.

The algorithm produces a "match score" as an output for each sample: this is the largest of the Pearson's correlations between the sample spectrum and each of the library spectra. The median score from correct identifications across all classes was 0.993. In contrast, the median score for the incorrect identifications was 0.96. A summary figure expanding on these values is given in the Supporting Information. It is worth noting that the median score for the amphetamine class was 0.993 and that this was also the individual score for the technical replicate of MDMA; this was the most prevalent compound amongst the seized samples discussed below.

For the 32 validation mixtures, there were 21 correct identifications of both compounds present in the sample. The median match score for these was 0.923 . This is substantially lower than the typical match scores for single compounds, reflecting the increased difficulty of identifying unknown binary mixtures of unknown concentration ratios. A further 10 were identified as single compounds instead of mixtures, with one of the two compounds being correctly identified. The final sample was found to be a mixture, but one of the individual compounds was incorrectly identified as a different cutting agent; importantly, therefore, this was not a false positive with regards to controlled substances. From consideration of the outcomes from the validation samples, a threshold was set empirically at 0.838: match scores greater than this are indicative of a correct match, whereas identifications with lower scores are considered unreliable.

Analysis of Seized Samples. 432 seized samples were analyzed as part of this work. These samples represent a seized material within the Greater Manchester region and tested by the MANchester DRug Analysis \& Knowledge Exchange (MANDRAKE) partnership in accordance with the UK Misuse of Drugs Act (1971) and Misuse of Drugs Regulations (2001). All samples were analyzed using ${ }^{1} \mathrm{H}$ NMR spectroscopy (ca. 5-10 mg of sample dissolved in $0.6 \mathrm{~mL}$ of deuterated DMSO) and GC-MS (see Supporting Information for method and sample preparation). The results from the ${ }^{1} \mathrm{H}$ NMR analysis were cross-referenced with GC-MS data acquired from the same sample. The GC-MS validation approach is outlined in the Supporting Information. Compounds identified through the work detailed herein are shown in Figure 1.

Of the 432 samples analyzed, thirteen (3\%) possessed no peaks in the GC-MS chromatogram. Of these, ${ }^{1} \mathrm{H}$ NMR analysis identified that adulterants such as lactose were present, which would require derivatization before they could be detected by GC-MS. Two samples were identified as containing lactose only (median match score 0.964) and another one as sucrose only (match score 0.968 ). Two further 
samples were indicated to be comprised of sodium gammahydroxybutyric acid with a median match score of 0.851 . The other eight samples possessed match scores below the identification threshold (i.e. lower than 0.838), and thus their identifications were considered unreliable.

Three samples returned a null result in both the ${ }^{1} \mathrm{H}$ NMR and GC-MS, that is, they consisted of no material that possessed a ${ }^{1} \mathrm{H}$ NMR signature, and no peak was observed in the GC-MS trace.

The other 416 samples resulted in partial or complete agreement between the ${ }^{1} \mathrm{H}$ NMR and the GC-MS outcomes. Of these 416 samples, the result from the ${ }^{1} \mathrm{H}$ NMR analysis matched the GC-MS result exactly in 387 cases (93\%), thirteen of which were identified as being binary mixtures by both GC-MS and ${ }^{1} \mathrm{H}$ NMR. Partial agreement was obtained for 25 (6\%) of samples surveyed, whilst the outcomes from just 4 samples (1\%) did not match at all. A partial match here means that either the ${ }^{1} \mathrm{H}$ NMR analysis or the GC-MS trace were missing one or more components than the other method detected.

By far the most common drug identified was MDMA, accounting for 171 instances (40\% of samples surveyed). Cocaine and ketamine (85 (20\%) and 74 (17\%) instances respectively) were the next most common. Samples found to contain mephedrone, paracetamol, benzocaine, ethylone, heroin, caffeine, diazepam, or cannabis accounted for a further 23 samples (5\% of samples surveyed). Figure 2 shows the percentage breakdown by class of single and binary component samples. A recent report from a UK festival onsite drug checking service reported that the three most common drugs

(a)
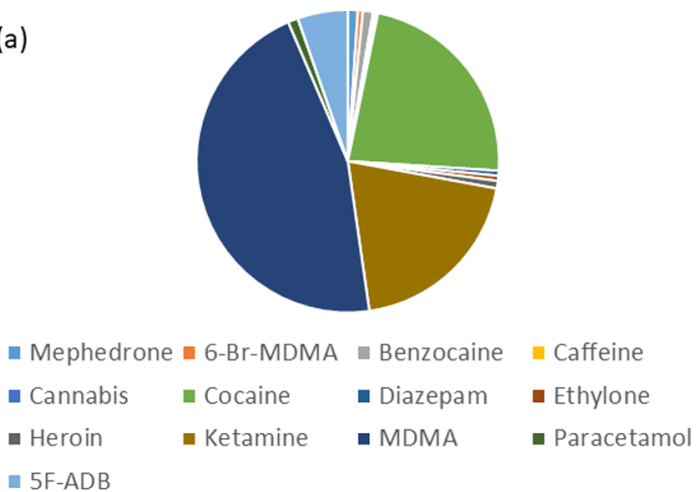

(b)

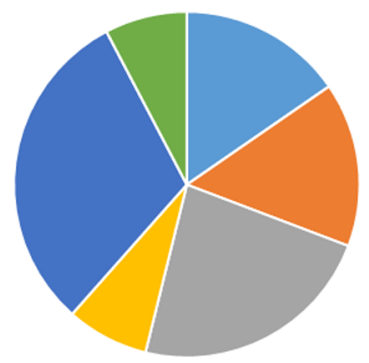

- Amphetamine and caffeine $\|$ Benzocaine and ketamine

- Cocaine and benzocaine $\quad$ Cocaine and caffeine

- Cocaine and levamisole $\quad$ Cocaine and phenacetin

Figure 2. Pie-chart showing the relative numbers of samples of different compositions, as identified by ${ }^{1} \mathrm{H}$ NMR analysis and subsequently validated by GC-MS, for (a) single component samples and (b) binary mixtures. identified were MDMA (57\%), ketamine (13.5\%), and cocaine $(10 \%) .{ }^{35}$ The results presented here reaffirm these drugs as the most commonly encountered and with similar frequencies.

The median match score for the seized MDMA samples was found to be 0.986 (range 0.858-0.997). This implies that some of the samples analyzed were almost of reference standard quality. In theory, an exact match with a reference compound will have a score of 1 ; in practice, sources of variance such as instrumental drift, spectral noise, and concentration differences mean that this is rarely achieved. The value obtained for the technical replicate of the reference MDMA discussed above was 0.993. Figure 3 shows the ${ }^{1} \mathrm{H}$ NMR spectral variation of ten seized samples containing MDMA in comparison to the ${ }^{1} \mathrm{H}$ NMR reference spectrum of MDMA.

Similarly, ketamine and cocaine samples had median match scores of 0.980 and 0.973 respectively. For the cocaine samples, the ${ }^{1} \mathrm{H}$ NMR analysis was able to differentiate between the free-base form and the hydrochloride salt. Four instances of free-base cocaine were found, for which the median match score was 0.184 higher than that of cocaine hydrochloride (median match-score for free-base cocaine was found to be 0.987 ). The difference between the two match scores is important, as the match score for cocaine hydrochloride is below the validation threshold in some instances. Although the ${ }^{1} \mathrm{H}$ NMR spectroscopy analysis reveals this difference, $\mathrm{GC}-\mathrm{MS}$ only reports the presence of cocaine.

It is noteworthy that MDMA and ketamine both possess amine protons and the ${ }^{1} \mathrm{H}$ NMR chemical shift is concentration-dependent (see Figure 3 for the variation of the amine protons of MDMA at ca. $\delta 9.1$ ). Thus, the algorithm developed was able to successfully deal with this and still match the ${ }^{1} \mathrm{H}$ NMR spectrum to that of the reference spectrum. The use of deuterated DMSO also prevents this amine proton from being deuterated, and thus the molecule is not needed to be chemically changed to facilitate its successful detection. The median match scores for the other single component samples are shown in Table 1.

The detection of 6-Br-MDMA in eight samples (two as the sole component and six as a binary mixture with magnesium stearate) highlights a way in which the method can be used to identify a drug class, despite the material not being in the reference library. Initial analysis of these eight samples indicated that the material was a possible unknown-all the match scores were below the validation threshold as they ranged from 0.755 to 0.644 . Five samples reported the sample consisting of an amphetamine (MDMA), whilst the other three were indicated to be $N$-ethylpentylone hydrochloride, or a precursor to its production, piperonal. Despite the range of materials suggested, there is commonality, in that all of the compounds possess a 1,2-methylenedioxybenzene ring, with the benzene ring possessing a substituted aliphatic chain or functional group. Analysis of the GC-MS traces for these eight compounds identified a single component that had a retention time of $6.7 \mathrm{~min}$. Subsequent analysis of the samples using highfield ${ }^{1} \mathrm{H}$ and ${ }^{13} \mathrm{C}$ NMR spectroscopy and MS in conjunction with previously reported ${ }^{36}$ MS data revealed the structure to be 6-Br-MDMA. A sample was synthesized and the reference library updated accordingly with its ${ }^{1} \mathrm{H}$ NMR spectrum. This enabled two of the samples to be identified as 6-Br-MDMA with a median match-score of 0.896, although a further reference sample needed to be created to correctly identify the 

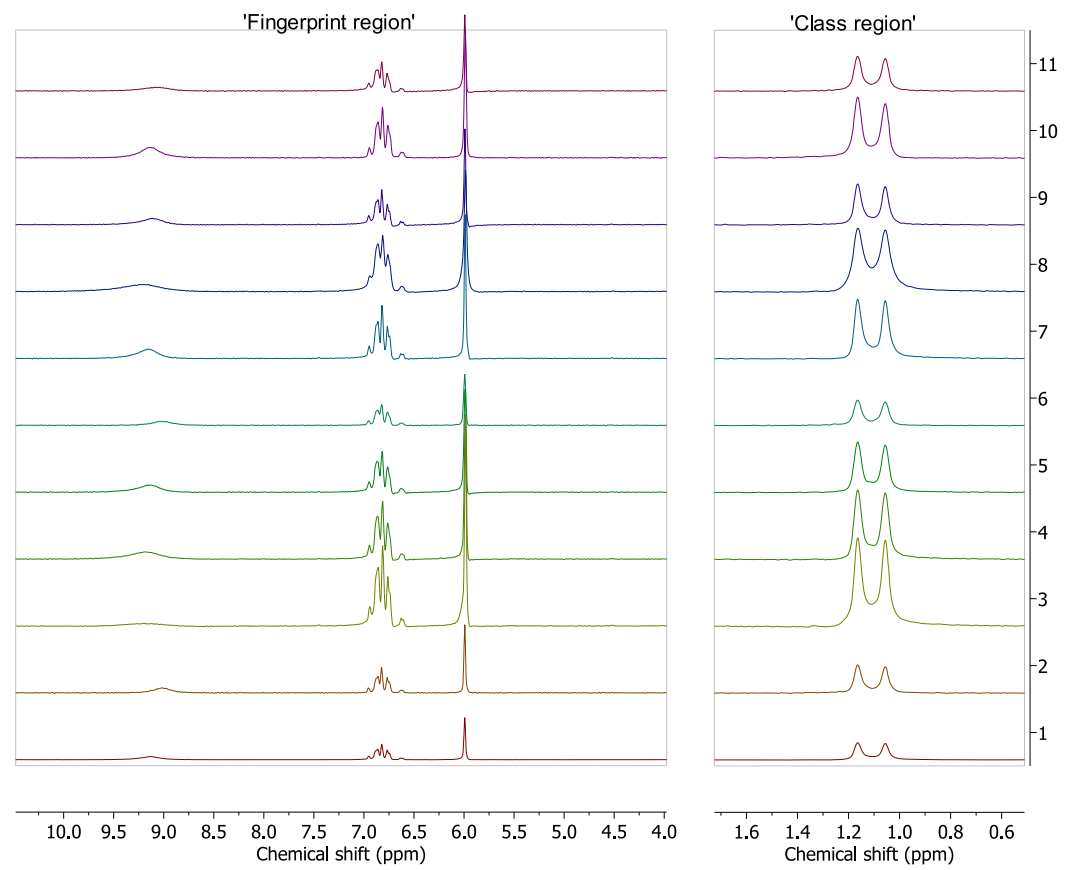

Figure 3. Series of ${ }^{1} \mathrm{H}$ NMR spectra of MDMA. Spectrum 1 is the reference spectrum that is included in the reference library, whereas spectra $2-$ 11 are seized samples (sample IDs 123, 207, 244, 309, 377, 405, 561B, 582, 598A, 598B, and 613).

Table 1. Median Match Scores for the Detection of Single Component Mixtures (Inclusive of Null Responses) ${ }^{a}$

$\begin{array}{lc}\text { compound detected } & \text { median match score } \\ \text { MDMA } & 0.986(171) \\ \text { 6-Br-MDMA } & 0.896(2) \\ \text { ketamine } & 0.980(74) \\ \text { cocaine } & 0.973(85) \\ \text { benzocaine } & 0.960(4) \\ \text { mephedrone } & 0.909(4) \\ \text { paracetamol } & 0.939(4) \\ \text { ethylone } & 0.939(2) \\ \text { heroin } & 0.858(3) \\ \text { caffeine } & 0.855(1) \\ \text { diazepam } & 0.959(2) \\ \text { cannabis } & 0.937(2) \\ \text { 5F-ADB } & 0.899(20)\end{array}$

${ }^{a_{T}}$ The number of samples identified of each type are indicated in parentheses.

other six, due to the presence of magnesium stearate. These samples are discussed further in the mixtures section.

The detection of diazepam in two samples required a modification of the general procedure to enable its analysis by ${ }^{1} \mathrm{H}$ NMR spectroscopy. In general benzodiazepines such as diazepam are produced as tablets which contain a large concentration of lactose. When part of the tablet is dissolved in DMSO both the API and excipient are soluble. The lactose is in such a high concentration that it swamps the signals from the diazepam. The subsequent result from matching the fingerprint is usually lactose. By contrast, the benzodiazepines are soluble in acetonitrile while the lactose is practically insoluble. Repeating the sample prep using deuterated acetonitrile in place of DMSO- $d_{6}$ allowing the ${ }^{1} \mathrm{H}$ NMR data acquisition and analysis to be performed under the same conditions as the other samples analyzed. The corresponding median match score for these two samples was 0.959 . There are other misused pharmaceuticals which may present a similar issue with the analysis, for example, buprenorphine. The procedure using deuterated acetonitrile is also applicable in these cases.

Of the 416 samples exhibiting GC-MS peaks, 24 samples were identified as synthetic cannabinoids, of which one was a mixture. The synthetic cannabinoids needed extracting from the plant material and were not seized as crystalline materials. All of the samples were typically less than $1.5 \%$ API by weight (see Supporting Information for details on the experimental protocol used to determine this) with only three samples possessing more than this threshold (highest 3.8\%). Twenty samples were found to comprise 5F-ADB as the sole API. We note here that some of match scores for the samples were very similar to other materials in the database. For example, four samples had match scores that differed by only $0.001-0.005$ compared to that of buprenorphine hydrochloride whilst another two samples were 0.003-0.008 different to MMBCHMINACA. A further three samples were indicated to be 5FADB by GC-MS but gave a null response in terms of the ${ }^{1} \mathrm{H}$ NMR analysis. The GC-MS traces for these three samples indicated that the API was $0.3-0.7 \%$. It is notable that of the four samples that did not produce a match between the GCMS result and that of the ${ }^{1} \mathrm{H}$ NMR analysis, three of them were synthetic cannabinoid samples.

Mixtures. The algorithm developed is capable of detecting binary mixtures, which is commonplace for drugs (e.g. cocaine) as they are "cut" with another material. Of the samples analyzed, thirteen samples were identified as being a binary mixture by both GC-MS and ${ }^{1} \mathrm{H}$ NMR, with a further 26 samples being identified as being a partial match, that is, one component not identified in the ${ }^{1} \mathrm{H}$ NMR spectrum when two or more are present in the GC-MS trace, or vice versa.

Of the thirteen binary mixtures correctly identified, $69 \%$ of these were cocaine based, the other $31 \%$ were amphetamine and caffeine (two instances) and benzocaine and ketamine (two instances). The cocaine samples were cut with caffeine, 
levamisole, benzocaine, or phenacetin. Four samples were identified as being a mixture of cocaine and levamisole by GC-MS (retention times 8.22 and 7.50 min respectively) and also by ${ }^{1} \mathrm{H}$ NMR analysis (median match score 0.885 ). Following analysis of the gas chromatogram, the amount of levamisole in these samples was observed to range from 3.6 to $17.6 \%$. The success of the ${ }^{1} \mathrm{H}$ NMR analysis is particularly noteworthy here, given that levamisole and cocaine have similar aromatic moieties and therefore overlap in the ${ }^{1} \mathrm{H}$ NMR spectrum. The class region is key to distinguishing between the two different components in these cases, whilst also facilitating the identification of both components present.

One instance of a cocaine and phenacetin mixture was identified by ${ }^{1} \mathrm{H}$ NMR (match score 0.837 ) and validated by the GC-MS data. Analysis of the gas chromatogram revealed that cocaine was prevalent in this mixture $(79.4 \%$ cocaine and $20.6 \%$ phenacetin). The relative amount of cocaine in the single instance of cocaine mixed with caffeine was $90.2 \%$. The match score for this sample was 0.922. A similar median match-score (0.849) was obtained for the two cocaine and benzocaine samples despite the composition of cocaine varying from 24.7 to $80.9 \%$.

Two samples were identified as amphetamine and caffeine $\left({ }^{1} \mathrm{H}\right.$ NMR median match score 0.890$)$. From GC analysis, the relative amount of amphetamine present ranged from 23.3 to $36.7 \%$. Similarly, the two samples that returned ketamine and benzocaine by both ${ }^{1} \mathrm{H}$ NMR and GC-MS analysis had a ${ }^{1} \mathrm{H}$ NMR median match score of 0.919 .

Partial Matches. 26 samples were identified as partial matches. Six of these that contained 6-Br-MDMA, in its hydrochloride form, were found to also consist of magnesium stearate. In the absence of 6-Br-MDMA, magnesium stearate does not dissolve in DMSO, as evidenced by the lack of peaks in the ${ }^{1} \mathrm{H}$ NMR spectrum. However, it was observed that if magnesium stearate was added to a sample of 6-Br-MDMA hydrochloride in DMSO, a new peak was observed at $1.21 \mathrm{ppm}$ in the ${ }^{1} \mathrm{H}$ NMR spectrum with concomitant loss of the amine peak at $9.23 \mathrm{ppm}$ (see Supporting Information for ${ }^{1} \mathrm{H}$ NMR spectra). It is believed that a disproportionation reaction had occurred, leading to the exchange of the chloride for stearate which resulted in magnesium chloride being formed. The practice of increasing the water solubility of drugs using disproportionation is well-documented. ${ }^{37-40}$ Some samples were more heavily cut with magnesium stearate than others, and thus a reference ${ }^{1} \mathrm{H}$ NMR spectrum of 6-Br-MDMA with magnesium stearate was required. A suitable sample was prepared, a ${ }^{1} \mathrm{H}$ NMR spectrum acquired, and the reference library was updated accordingly. Subsequently, the median match score for the six seized samples was 0.926 . These were recorded as partial matches because magnesium stearate cannot be observed under the GC-MS conditions employed and so could not be validated.

Ten cocaine binary mixtures were recorded as partial matches, with a further three that were found to be tertiary mixtures and one that comprised six components. One of the tertiary mixtures by GC-MS proved to comprise benzocaine, paracetamol, and cocaine in a ratio of 6.6:15.8:77.6. The ${ }^{1} \mathrm{H}$ NMR analysis, which is limited to reporting on binary mixtures only, returned paracetamol and cocaine as the match, with a match score of 0.926 . Benzocaine was not reported, most likely due to the low concentration present in the sample. Similarly, another tertiary mixture was identified to be cocaine, benzocaine, and levamisole by GC-MS and returned cocaine and benzocaine as the match in the ${ }^{1} \mathrm{H}$ NMR analysis with a match score of 0.869 . As levamisole is only present at a level of $0.4 \%$, it is not surprising that the ${ }^{1} \mathrm{H}$ NMR analysis identified the two most prevalent components. The final tertiary mixture was found to consist of cocaine, ketamine, and benzocaine in a 35.6:35.7:28.7 ratio. The match scores for benzocaine and cocaine, and benzocaine and ketamine, differ by only 0.007 for this sample being 0.856 and 0.849 , respectively. Thus, despite the algorithm being limited to detecting two components only, appraisal of the match scores can provide evidence for the presence of three components in a mixture.

The six-component mixture consisted of cocaine, levamisole, hexadecanoic acid, tolycaine, heptan-3-ol, and phenacetin with cocaine $(87.9 \%)$ and phenacetin $(7.4 \%)$ being the most prevalent. The ${ }^{1} \mathrm{H}$ NMR analysis reported cocaine and phenacetin with a match-score of 0.862 . It is noteworthy that the other components were all present at a level of $3 \%$ or lower, and this will have hindered their detection in the ${ }^{1} \mathrm{H}$ NMR spectrum.

Of the ten cocaine binary mixtures, three samples did not return cocaine as the primary identified API. All of these samples comprised largely of benzocaine (>79\%), and hence, the ${ }^{1} \mathrm{H}$ NMR spectrum was dominated by the signals of benzocaine. Furthermore, the lower molecular weight of benzocaine compared to cocaine results in lower signal intensity of the latter in the ${ }^{1} \mathrm{H}$ NMR spectrum, also hindering its detection.

A further six samples were cocaine and levamisole binary mixtures. For five of these samples, levamisole comprised $<2 \%$ of the sample according to GC-MS analysis (with one exception where the levamisole was present at the $5 \%$ level), and this might have prevented its detection in the ${ }^{1} \mathrm{H}$ NMR analysis. The other sample indicated levamisole as an additional component in the ${ }^{1} \mathrm{H}$ NMR analysis. Although this represents a false-positive hit, the important aspect is that cocaine is detected in the sample as this is the main API. The final cocaine containing sample was shown to be cut with caffeine but only at the level of $1 \%$. Again, this low amount is not conducive to its detection by ${ }^{1} \mathrm{H}$ NMR spectroscopy.

The remaining six samples were indicated by ${ }^{1} \mathrm{H}$ NMR to have one too few or one too many components compared to the GC-MS trace. One of these samples returned only AMBFUBINACA by ${ }^{1} \mathrm{H}$ NMR whilst the GC-MS showed it comprised the former as well as $5 \mathrm{~F}-\mathrm{ADB}$. Both components on a weight-by-weight basis were $<2.5 \%$, with AMB-FUBINACA being present at almost five times the amount of 5F-ADB. Another sample was shown to comprise alpha-D-lactose and caffeine whereas GC-MS reported only caffeine; this is as expected, as lactose would need to be derivatized in order to be detectable under the GC conditions employed. Another sample of note here is one that comprised methiopropamine and 2 -aminoindane by GC-MS but was determined to be methiopropamine and amphetamine sulfate following ${ }^{1} \mathrm{H}$ NMR analysis. Despite the difference in compositions, it must be noted that 2 -aminoindane is the rigid analogue of amphetamine and hence has similar ${ }^{1} \mathrm{H}$ NMR resonances; thus there is a scientific rationale for its detection following ${ }^{1} \mathrm{H}$ NMR analysis.

\section{CONCLUSIONS}

There is a need for a high-throughput analytical technique with facile sample preparation to deal with increasing numbers of drug seizures made by law agencies. This paper presents an 
approach comprising an automated NMR benchtop spectrometer, which acquires, processes, and interprets the spectral data with minimum user knowledge. The findings of the analysis are reported in around $5 \mathrm{~min}$.

The approach makes use of an algorithm that analyses the acquired ${ }^{1} \mathrm{H}$ NMR spectrum by matching it to a library of over 300 reference spectra from known substances. 432 seized samples were tested using this system, and the outcomes compared with GC-MS data, a currently accepted reference method (see Table 2). The GC-MS trace in thirteen cases

Table 2. Analysis Breakdown for the 432 Samples Surveyed as Part of This Work ${ }^{a}$

$$
\begin{array}{ll}
\text { no. of samples } & 432 \\
\% \text { of samples that could not be validated } & 3.0(13) \\
\text { \% samples found to contain no API or cutting agent } & 0.7(3) \\
\text { samples that do not match } & 0.9(4) \\
\% \text { correctly matched (single component mixtures) } & 86.6(374) \\
\% \text { correctly matched (two-component samples) } & 3.0(13) \\
\% \text { partially matched (two or more component samples) } & 5.8(25)
\end{array}
$$

${ }^{a}$ The number in parentheses indicate the number of samples of that type.

(3\% of the samples surveyed) were devoid of any peaks, so the ${ }^{1} \mathrm{H}$ NMR analysis could not be validated for these samples. A further three samples were found to lack any API or cutting agent after both ${ }^{1} \mathrm{H}$ NMR and GC-MS analysis.

For the remaining 416 samples, the outcomes by GC-MS and ${ }^{1} \mathrm{H}$ NMR matched in $93 \%$ of cases. Of these, thirteen samples were identified as being a binary mixture by both GC-MS and ${ }^{1} \mathrm{H}$ NMR. A partial match (one component not identified) was obtained for $6 \%$ of samples surveyed, whilst only $1 \%$ of samples resulted in contradictory outcomes.

The analytical predictive power of the algorithm was exemplified in the detection of 6-Br-MDMA which identified the methylenedioxy ring structure and the class of compound. Further characterization of the samples, and the synthesis of a reference sample, led to the complete elucidation of the chemical structure. Note that the disproportionation of chloride for stearate from magnesium stearate present in the majority of seized samples of this type required an additional reference ${ }^{1} \mathrm{H}$ NMR spectrum to be acquired and the spectral library updated accordingly. Eight samples were found to possess 6-Br-MDMA as the API, with six of these being heavily cut with magnesium stearate, as indicated by the match scores.

The protocol was modified for the detection of benzodiazepines, for example, diazepam. Deuterated acetonitrile was used instead, but the methodology for detection, acquisition, processing, and reporting remained consistent with that of the samples collected in deuterated DMSO.

Having demonstrated the validity of the approach, future work will be directed toward a quantification method to widen the applicability of the qualitative approach employed here, and to improve the ability to handle multicomponent mixtures.

\section{METHODOLOGY}

Materials. 302 reference compounds were synthesized (see below) or obtained either from Fluorochem (UK), Sigma Aldrich (UK), BRC Fine Chemicals (https://www.brcfinechemicals.com/ - prior to the UK Psychoactive Substances Act (2016), which came in to effect on the 26th May 2016 and made it an offence to manufacture, supply, possess, and import and export psychoactive substances named in this Act) or Carbosynth (UK). The following groups of chemicals were synthesized according to, or adapted from, the literature references cited: 4-methoxyamphetamines, ${ }^{41} 2$-, 3 - and 4fluoroamphetamines, ${ }^{42}$ thiopropamine, ${ }^{43}$ methiopropamine, ${ }^{44}$ dimethyldiphenidines, ${ }^{23} 3$ - and 4-chloroethcathinone, ${ }^{45}$ trifluoromethylmethcathinones, ${ }^{45} 3$ - and 4-chloro $\mathrm{N}, \mathrm{N}$-diethylcathinones, ${ }^{46} 2-$, 3- and 4-methcathinones, ${ }^{46}$ 2-, 3- and 4fluoromethcathinones, ${ }^{47}$ diphenidine and ephenidine derivatives, ${ }^{23}$ fentanyl derivatives, ${ }^{48}$ fluorolintanes, ${ }^{23}$ 25I-,25B-, 25C-, and 25F-NBOMe, ${ }^{49}$ MMB-CHMINACA, MMB-CHMICA, 5F-ADB, AMB-FUBINACA, and AB-FUBINACA, ${ }^{50}$ 2C-C, 2C-B, 2C-I, 2C-F, and 2C-H. ${ }^{51}$ All synthesized reference standards were fully characterized and exhibited physical and spectral data consistent with the literature and determined to be $>99 \%$ pure by high-field NMR. These consisted of a range of narcotics, NPS, and controlled substances, as well as commonly encountered non-controlled substances and adulterants. In total, there were 21 classes of compound: aminoindanes, amphetamines, atypical diphenidines, benzodiazepines, cathinones, diphenidines, ephenidines, fentanyls, fluorolintanes, methylenedioxycathinones, NBOMe, narcotics, pesticides, pharmaceuticals, phenethylamines, piperazines (including MT-45 and its derivatives), precursors, steroids, and synthetic cannabinoids. Two further "classes" of noncontrolled substances were also included: a range of "cutting agents" and "background residues" (i.e., only DMSO).

432 seized samples of unknown identity were obtained from Greater Manchester Police via the MANchester DRug Analysis \& Knowledge Ex-change (MANDRAKE) partnership and were stored and analyzed in accordance with the UK Misuse of Drugs Act (1971) and Misuse of Drugs Regulations (2001). The samples were collected over the period January 2017August 2018. All of these samples were supplied in their solid, bulk forms. For the seized materials, a micro-spatula tip of the material (ca. 5-10 mg) was dissolved in $0.6 \mathrm{~mL}$ of deuterated DMSO. If diazepam was suspected, then $0.6 \mathrm{~mL}$ of deuterated acetonitrile was used instead. For spice samples, approximately $50 \mathrm{mg}$ of material was weighed out and $0.6 \mathrm{~mL}$ of deuterated DMSO added. The spice material was heavily agitated to ensure that the API became dissolved in the DMSO. All samples were filtered through a $0.45 \mu \mathrm{m}$ polyvinylidene difluoride syringe filter (Whatman) directly into a NMR tube. Samples were analyzed by ${ }^{1} \mathrm{H}$ NMR and validated against contemporaneously acquired GC-MS data (GC-MS method outlined in Supporting Information).

The reference samples were prepared for NMR analysis by dissolving $10 \mathrm{mg}$ of compound per $1 \mathrm{~mL}$ of deuterated DMSO, or in the case of diazepam, deuterated MeCN.

130 compounds from the reference collection were available in sufficient quantity to prepare a replicate sample. All but three of the controlled substance classes (NBOMe, 2Cderivatives and piperazines) were represented in this validation collection. A further 32 samples of non-controlled substances in the reference collection were prepared in pairwise combinations, to assist with development of the mixture identification method.

NMR Data Acquisition. ${ }^{1} \mathrm{H}$ NMR spectra were acquired of all samples using a Pulsar benchtop NMR spectrometer (Oxford Instruments, Abingdon, UK) operating at a frequency of $59.7 \mathrm{MHz}$. The temperature of the probe was calculated to be $308.5 \mathrm{~K}$ by measuring the separation (in $\mathrm{Hz}, \Delta \delta$ ) between the $\mathrm{CH}_{2}$ and $\mathrm{OH}$ signals of neat ethylene glycol and 
implementing the equation $T[\mathrm{~K}]=466.5-102.00 \Delta \delta .^{52}$ After the sample had been inserted, an automated procedure began whereby the instrument would lock on to the deuterated signature of DMSO (thus used as a chemical shift reference) before acquiring 16 scans (for the spice samples, 64 scans were acquired).

Following acquisition, the data were processed in MNova (Mestrelab Research, Santiago de Compostela, Spain) using an automated script file. The processed FID was then analyzed by the pattern recognition algorithm, developed in-house using Matlab (The Mathworks Inc, Cambridge, UK). The algorithm employs a minimum distance classifier. The multivariate distance between the sample spectrum and each of the reference spectra is calculated. The sample is identified as the nearest reference compound, provided the "match score" (equal to one minus the distance) exceeds an (empirically determined) threshold; if it does not, then the outcome is considered to be tentative, unreliable, or unknown. Binary mixtures are accommodated by extending the pattern search with synthetically generated mixture spectra of pairwise combinations of the reference library.

The collection of NMR data and the subsequent analysis takes approximately $5 \mathrm{~min}$. If signal-to-noise was found to be poor, more scans were obtained. This was only required in less than $1 \%$ of the samples being analyzed, and this was due to the amount of sample available being low, for example, the residue material within plastic packets used to transport illicit materials.

\section{ASSOCIATED CONTENT}

\section{S Supporting Information}

The Supporting Information is available free of charge on the ACS Publications website at DOI: 10.1021/acsomega.9b00302.

GC-MS method employed to screen samples, median match scores of correction classifications of validation spectra, tables detailing the result of ${ }^{1} \mathrm{H}$ NMR and GCMS analysis for all samples and ${ }^{1} \mathrm{H}$ NMR spectra showing the effect of adding magnesium stearate to 6-BrMDMA (PDF)

\section{AUTHOR INFORMATION}

\section{Corresponding Authors}

*E-mail: David.Williamson@oxinst.com. Phone: +44 (0) 1865 393200 (D.C.W.).

*E-mail: Kate.Kemsley@quadram.ac.uk. Phone: +44 (0)1603 255000 (E.K.K.).

*E-mail: O.Sutcliffe@mmu.ac.uk. Phone: +44 (0)161 2471531 (O.B.S.).

*E-mail: R.Mewis@mmu.ac.uk. Phone: +44 (0)161 2471432 (R.E.M.).

\section{ORCID}

E. Kate Kemsley: 0000-0003-0669-3883

Oliver B. Sutcliffe: 0000-0003-3781-7754

Ryan E. Mewis: 0000-0002-3756-6505

\section{Present Address}

${ }^{\#}$ Forensic Drug Research Centre, Centre for Anatomy and Human Identification, School of Science and Engineering, University of Dundee, Dundee, DD1 5EH, UK.

\section{Author Contributions}

The manuscript was written through contributions of all authors.

Notes

The authors declare no competing financial interest.

\section{ACKNOWLEDGMENTS}

O.B.S. and R.E.M. wish to thank Manchester Metropolitan University and Oxford Instruments for the provision of matchfunded studentships for J.M. and M.C.H. Manchester Metropolitan University is also thanked for a Vice-Chancellor Studentship for T.B.R.R. The Natural Sciences and Engineering Research Council of Canada (396154510) and Fonds de Recherche du Québec-Nature et Technologie (206375) are thanked for funding N.G.

\section{REFERENCES}

(1) Bicknell, V.; Gerostamoulos, D.; Ranson, D. Novel Psychoactive Substances: the challenges for health care, analytical science and the law. J. Law. Med. 2017, 24, 763-766.

(2) Graddy, R.; Buresh, M. E.; Rastegar, D. A. New and Emerging Illicit Psychoactive Substances. Med. Clin. North Am. 2018, 102, 697714.

(3) Baumeister, D.; Tojo, L. M.; Tracy, D. K. Legal highs: staying on top of the flood of novel psychoactive substances. Ther. Adv. Psychopharmacol. 2015, 5, 97-132.

(4) European Drug Report 2015. Trends and Developments; EMCDDA: Luxembourg, 2015.

(5) European Drug Report. Trends and Developments; EMCDDA: Luxembourg, 2018.

(6) Harper, L.; Powell, J.; Pijl, E. M. An overview of forensic drug testing methods and their suitability for harm reduction point-of-care services. Harm. Redcut. J. 2017, 14, 1.

(7) Pereira, L. S. A.; Lisboa, F. L. C.; Neto, J. C.; Valladão, F. N.; Sena, M. M. Direct classification of new psychoactive substances in seized blotter papers by ATR-FTIR and multivariate discriminant analysis. Microchem. J. 2017, 133, 96-103.

(8) Pereira, L. S. A.; Lisboa, F. L. C.; Coelho Neto, J.; Valladão, F. N.; Sena, M. M. Screening method for rapid classification of psychoactive substances in illicit tablets using mid infrared spectroscopy and PLS-DA. Forensic Sci. Int. 2018, 288, 227-235.

(9) McLaughlin, G.; Morris, N.; Kavanagh, P. V.; Power, J. D.; O'Brien, J.; Talbot, B.; Elliott, S. P.; Wallach, J.; Hoang, K.; Morrish, H.; Brandti, S. D. Test purchase, synthesis, and characterization of 2methoxydiphenidine (MXP) and differentiation from its meta- and para-substituted isomers. Drug Test. Anal. 2016, 8, 98-109.

(10) Apirakkan, O.; Frinculescu, A.; Shine, T.; Parkin, M. C.; Cilibrizzi, A.; Frascione, N.; Abbate, V. Analytical characterization of three cathinone derivatives, 4-MPD, 4F-PHP and bk-EPDP, purchased as bulk powder from online vendors. Drug Test. Anal. 2018, 10, 372-378.

(11) Fikiet, M. A.; Khandasammy, S. R.; Mistek, E.; Ahmed, Y.; Halámková, L.; Bueno, J.; Lednev, I. K. Surface enhanced Raman spectroscopy: A review of recent applications in forensic science. Spectrochim. Acta, Part A 2018, 197, 255-260.

(12) Khandasammy, S. R.; Fikiet, M. A.; Mistek, E.; Ahmed, Y.; Halámková, L.; Bueno, J.; Lednev, I. K. Bloodstains, paintings, and drugs: Raman spectroscopy applications in forensic science. Forensic Chem. 2018, 8, 111-133.

(13) Penido, C. A. F. d. O.; Pacheco, M. T. T.; Lednev, I. K.; Silveira, L. Raman spectroscopy in forensic analysis: identification of cocaine and other illegal drugs of abuse. J. Raman Spectrosc. 2016, 47, $28-38$.

(14) Elie, L.; Elie, M.; Cave, G.; Vetter, M.; Croxton, R.; Baron, M. Microcrystalline testing used in combination with Raman microspectroscopy for absolute identification of novel psychoactive substances. J. Raman Spectrosc. 2016, 47, 1343-1350. 
(15) Gabrielli, L.; Rosa-Gastaldo, D.; Salvia, M.-V.; Springhetti, S.; Rastrelli, F.; Mancin, F. Detection and identification of designer drugs by nanoparticle-based NMR chemosensing. Chem. Sci. 2018, 9, $4777-4784$.

(16) Zivkovic, A.; Bandolik, J. J.; Skerhut, A. J.; Coesfeld, C.; Prascevic, M.; Zivkovic, L.; Stark, H. Quantitative Analysis of Multicomponent Mixtures of Over-the Counter Pain Killer Drugs by Low-Field NMR Spectroscopy. J. Chem. Educ. 2017, 94, 121-125.

(17) Ernst, L.; Brandhorst, K.; Papke, U.; Altrogge, A.; Zodel, S.; Langer, N.; Beuerle, T. Identification and quantification of synthetic cannabinoids in 'spicelike' herbal mixtures: Update of the German situation in early 2017. Forensic Sci. Int. 2017, 277, 51-58.

(18) Lowdon, J. W.; Alkirkit, S. M. O.; Mewis, R. E.; Fulton, D.; Banks, C. E.; Sutcliffe, O. B.; Peeters, M. Engineering molecularly imprinted polymers (MIPs) for the selective extraction and quantification of the novel psychoactive substance (NPS) methoxphenidine and its regioisomers. Analyst 2018, 143, 2002-2007.

(19) Maher, S.; Elliott, S. P.; George, S. The analytical challenges of cyclopropylfentanyl and crotonylfentanyl: An approach for toxicological analysis. Drug Test. Anal. 2018, 10, 1483-1487.

(20) Boateng, B. O.; Fever, M.; Edwards, D.; Petersson, P.; Euerby, M. R.; Sutcliffe, O. B. Chromatographic retention behaviour, modelling and optimization of a UHPLC-UV separation of the regioisomers of the Novel Psychoactive Substance (NPS) methoxphenidine (MXP). J. Pharm. Biomed. Anal. 2018, 153, 238-247.

(21) Poplawska, M.; Blazewicz, A.; Kaminski, K.; Bednarek, E.; Fijalek, Z.; Kozerski, L. Application of high-performance liquid chromatography with charged aerosol detection (LC-CAD) for unified quantification of synthetic cannabinoids in herbal blends and comparison with quantitative NMR results. Forensic Toxicol. 2018, 36, 122-140.

(22) Mercieca, G.; Odoardi, S.; Cassar, M.; Strano Rossi, S. Rapid and simple procedure for the determination of cathinones, amphetamine-like stimulants and other new psychoactive substances in blood and urine by GC-MS. J. Pharm. Biomed. Anal. 2018, 149, 494-501.

(23) Geyer, P. M.; Hulme, M. C.; Irving, J. P. B.; Thompson, P. D.; Ashton, R. N.; Lee, R. J.; Johnson, L.; Marron, J.; Banks, C. E.; Sutcliffe, O. B. Guilty by dissociation-development of gas chromatography-mass spectrometry (GC-MS) and other rapid screening methods for the analysis of 13 diphenidine-derived new psychoactive substances (NPSs). Anal. Bioanal. Chem. 2016, 408, 8467-8481.

(24) Baron, M.; Elie, M.; Elie, L. An analysis of legal highs - do they contain what it says on the tin? Drug Test. Anal. 2011, 3, 576-581.

(25) Elie, M. P.; Elie, L. E.; Baron, M. G. Keeping pace with NPS releases: fast GC-MS screening of legal high products. Drug Test. Anal. 2013, 5, 281-290.

(26) Jones, L. E.; Stewart, A.; Peters, K. L.; McNaul, M.; Speers, S. J.; Fletcher, N. C.; Bell, S. E. J. Infrared and Raman screening of seized novel psychoactive substances: a large scale study of $>200$ samples. Analyst 2016, 141, 902-909.

(27) Guirguis, A.; Girotto, S.; Berti, B.; Stair, J. L. Identification of new psychoactive substances (NPS) using handheld Raman spectroscopy employing both 785 and 1064 nm laser sources. Forensic Sci. Int. 2017, 273, 113-123.

(28) Penido, C. A. F. d. O.; Pacheco, M. T. T.; Novotny, E. H.; Lednev, I. K.; Silveira, L. Quantification of cocaine in ternary mixtures using partial least squares regression applied to Raman and Fourier transform infrared spectroscopy. J. Raman Spectrosc. 2017, 48, 17321743.

(29) Causanilles, A.; Kinyua, J.; Ruttkies, C.; van Nuijs, A. L. N.; Emke, E.; Covaci, A.; de Voogt, P. Qualitative screening for new psychoactive substances in wastewater collected during a city festival using liquid chromatography coupled to high-resolution mass spectrometry. Chemosphere 2017, 184, 1186-1193.

(30) Lian, R.; Wu, Z.; Lv, X.; Rao, Y.; Li, H.; Li, J.; Wang, R.; Ni, C.; Zhang, Y. Rapid screening of abused drugs by direct analysis in real time (DART) coupled to time-of-flight mass spectrometry (TOFMS) combined with ion mobility spectrometry (IMS). Forensic Sci. Int. 2017, 279, 268-280.
(31) Seither, J. Z.; Hindle, R.; Arroyo-Mora, L. E.; DeCaprio, A. P. Systematic analysis of novel psychoactive substances. I. Development of a compound database and HRMS spectral library. Forensic Chem. 2018, 9, 12-20.

(32) Zhong, Y.; Huang, K.; Luo, Q.; Yao, S.; Liu, X.; Yang, N.; Lin, C.; Luo, X. The Application of a Desktop NMR Spectrometer in Drug Analysis. Int J Anal Chem 2018, 2018-3104569. DOI: 10.1155/ 2018/3104569

(33) Duffy, J.; Urbas, A.; Niemitz, M.; Lippa, K.; Marginean, I. Differentiation of fentanyl analogues by low-field NMR spectroscopy. Anal. Chim. Acta 2019, 1049, 161-169.

(34) Assemat, G.; Dubocq, F.; Balayssac, S.; Lamoureux, C.; MaletMartino, M.; Gilard, V. Screening of "spice" herbal mixtures: From high-field to low-field proton NMR. Forensic Sci. Int. 2017, 279, 8895.

(35) Measham, F. C. Drug safety testing, disposals and dealing in an English field: Exploring the operational and behavioural outcomes of the UK's first onsite 'drug checking' service. Int. J. Drug Policy 2018, pii, S0955.

(36) Liu, C.; Jia, W.; Qian, Z.; Li, T.; Hua, Z. Identification of five substituted phenethylamine derivatives 5-MAPDB, 5-AEDB, MDMA methylene homolog, 6-Br-MDMA, and 5-APB-NBOMe. Drug Test. Anal. 2017, 9, 199-207.

(37) John, C. T.; Xu, W.; Lupton, L. K.; Harmon, P. A. Formulating Weakly Basic $\mathrm{HCl}$ Salts: Relative Ability of Common Excipients to Induce Disproportionation and the Unique Deleterious Effects of Magnesium Stearate. Pharm. Res. 2013, 30, 1628-1641.

(38) Patel, M. A.; Luthra, S.; Shamblin, S. L.; Arora, K. K.; Krzyzaniak, J. F.; Taylor, L. S. Effect of excipient properties, water activity, and water content on the disproportionation of a pharmaceutical salt. Int. J. Pharm. 2018, 546, 226-234.

(39) Hirsh, D. A.; Su, Y.; Nie, H.; Xu, W.; Stueber, D.; Variankaval, N.; Schurko, R. W. Quantifying Disproportionation in Pharmaceutical Formulations with $35 \mathrm{Cl}$ Solid-State NMR. Mol. Pharm. 2018, 15, 4038-4048.

(40) Lee, G. S. H.; Craig, D.; Kannangara, G.; Dawson, M.; Conn, C.; Robertson, J.; Wilson, M. Analysis of 3,4-Methylenedioxy-NMethylamphetamine (MDMA) in "Ecstasy" Tablets by 13C Solid State Nuclear Magnetic Resonance (NMR) Spectroscopy. J. Forensic Sci. 1999, 44, 14550J.

(41) Liu, J.-T.; Sun, M.-P.; Tsai, Y.-S. Regioisomeric differentiation of mono-methoxy ring-substituted amphetamine and methamphetamine by GC-MS. Forensic Sci. Int. 2003, 2, 59-68.

(42) Plenevaux, A.; Dewey, S. L.; Fowler, J. S.; Guillaume, M.; Wolf, A. P. Synthesis of (R)-(-)-4-fluorodeprenyl and (S)-(+)-4-fluorodeprenyl and (R)-(-)-4-fluorodeprenyl and (S)-(+)-N-C-11-methyl-4fluorodeprenyl and positron emission tomography studies in baboon brain. J. Med. Chem. 1990, 33, 2015-2019.

(43) Campaigne, E.; McCarthy, W. C. 3-Substituted Thiophenes. VIII.1 3-Thienylalkylamines2. J. Am. Chem. Soc. 1954, 76, 44664468

(44) Blicke, F. F.; Burckhalter, J. H. $\alpha$-Thienylaminoalkanes. J. Am. Chem. Soc. 1942, 64, 477-480.

(45) Khreit, O. I. G.; Irving, C.; Schmidt, E.; Parkinson, J. A.; Nic Daeid, N.; Sutcliffe, O. B. Synthesis, full chemical characterisation and development of validated methods for the quantification of the components found in the evolved "legal high" NRG-2. J. Pharm. Biomed. Anal. 2012, 61, 122-135.

(46) Santali, E. Y.; Cadogan, A.-K.; Daeid, N. N.; Savage, K. A.; Sutcliffe, O. B. Synthesis, full chemical characterisation and development of validated methods for the quantification of $(+/-)-4$ '-methylmethcathinone (mephedrone): A new "legal high. J. Pharm. Biomed. Anal. 2011, 56, 246-255.

(47) Archer, R. P. Fluoromethcathinone, a new substance of abuse. Forensic Sci. Int. 2009, 185, 10-20.

(48) Valdez, C. A.; Leif, R. N.; Mayer, B. P. An Efficient, Optimized Synthesis of Fentanyl and Related Analogs. PLoS One 2014, 9, No. e108250. 
(49) Hansen, M.; Phonekeo, K.; Paine, J. S.; Leth-Petersen, S.; Begtrup, M.; Bräuner-Osborne, H.; Kristensen, J. L. Synthesis and Structure-Activity Relationships of N-Benzyl Phenethylamines as 5HT2A/2c Agonists. ACS Chem. Neurosci. 2014, 5, 243-249.

(50) Banister, S. D.; Moir, M.; Stuart, J.; Kevin, R. C.; Wood, K. E.; Longworth, M.; Wilkinson, S. M.; Beinat, C.; Buchanan, A. S.; Glass, M.; Connor, M.; McGregor, I. S.; Kassiou, M. Pharmacology of Indole and Indazole Synthetic Cannabinoid Designer Drugs ABFUBINACA, ADB-FUBINACA, AB-PINACA, ADB-PINACA, 5FAB-PINACA, 5F-ADB-PINACA, ADBICA, and 5F-ADBICA. ACS Chem. Neurosci. 2015, 6, 1546-1559.

(51) Shulgin, A.; Shulgin, A. PiHKAL: A Chemical Love Story; Transform Press: USA, 1991.

(52) Ammann, C.; Meier, P.; Merbach, A. A simple multi-nuclear NMR thermometer. J. Magn. Reson. 1982, 46, 319-321. 